\title{
Suppression of hepatocellular carcinoma cell proliferation by short hairpin RNA of frizzled 2 with Sonazoid-enhanced irradiation
}

\author{
MINORU TOMIZAWA ${ }^{1}$, FUMINOBU SHINOZAKI ${ }^{2}$, YASUFUMI MOTOYOSHI ${ }^{3}$, \\ TAKAO SUGIYAMA ${ }^{4}$, SHIGENORI YAMAMOTO ${ }^{5}$ and NAOKI ISHIGE ${ }^{6}$ \\ Departments of ${ }^{1}$ Gastroenterology, ${ }^{2}$ Radiology, ${ }^{3}$ Neurology, ${ }^{4}$ Rheumatology, ${ }^{5}$ Pediatrics and ${ }^{6}$ Neurosurgery, \\ National Hospital Organization, Shimoshizu Hospital, Yotsukaido City, Chiba 284-0003, Japan
}

Received October 1, 2015; Accepted November 5, 2015

DOI: 10.3892/ijo.2015.3259

\begin{abstract}
Short-hairpin RNA of frizzled-2 (shRNA-Fz2) is known to suppress the proliferation of hepatocellular carcinoma (HCC) cells; however, its effect on HCC cell motility is unknown. In this study, suppression of HCC cell motility by shRNA-Fz2 was analyzed, and introduction of shRNA-Fz2 into HCC cells was facilitated with ultrasound (US) irradiation generated from a diagnostic US device, which was enhanced by the contrast-enhanced US reagent Sonazoid. The HCC cell lines HLF and PLC/PRF/5 that were transfected with shRNA-Fz2 were plated to form monolayers, following which the cell monolayers were scratched with a sterile razor. After $48 \mathrm{~h}$, the cells were stained with hematoxylin and eosin, and the distance between the growing edge of the cell layer and the scratch lines was measured. Total RNA from the cells was isolated and subjected to real-time quantitative PCR to quantify matrix metalloproteinase 9 expression at $48 \mathrm{~h}$ after transfection of shRNA-Fz2. Starch-iodide method was applied to analyze the generation of $\mathrm{H}_{2} \mathrm{O}_{2}$ following US irradiation with the addition of Sonazoid in the liquid, and cell proliferation was analyzed $72 \mathrm{~h}$ later. The distances between the growing edge of the cell layer and the scratch lines and MMP9 expression levels were significantly decreased with transfection of shRNA-Fz2 $(\mathrm{P}<0.05)$. In the starch-iodide method, absorbance significantly decreased with the addition of Sonazoid $(\mathrm{P}<0.05)$, which suggested that US irradiation with Sonazoid generated $\mathrm{H}_{2} \mathrm{O}_{2}$ and enhanced sonoporation. ShRNA-Fz2 suppressed cell proliferation of both cell lines at a mechanical index of 0.4 Motility of HLF cells and PLC/PRF/5 cells was suppressed
\end{abstract}

Correspondence to: Dr Minoru Tomizawa, Department of Gastroenterology, National Hospital Organization, Shimoshizu Hospital, 934-5 Shikawatashi, Yotsukaido City, Chiba 284-0003, Japan

E-mail: nihminor-cib@umin.ac.jp

Key words: scratch assay, matrix metalloproteinase, starch-iodide method, cyclin D1, perfluorobutane microbubbles by shRNA-FZ2. Sonazoid enhanced sonoporation of the cells with the diagnostic US device and the suppression of proliferation of both HCC cell lines by shRNA-Fz2.

\section{Introduction}

Hepatocellular carcinoma (HCC) has a high incidence rate worldwide (1). It is treated with local ablation, surgery, transcatheter arterial chemoembolization, and systemic chemotherapy (2). Despite the advances in therapy, prognosis of HCC remains poor (3). Hence, molecular therapy is an emerging trend in the treatment of HCC (4).

The Wnt pathway is involved in the carcinogenesis in HCC (5). Without stimulation, Axin, Dishevelled (DVL), and glycogen synthase kinase-3 $\beta$ form a complex and degrade $\beta$-catenin (6). Wnt proteins bind to their receptor, frizzled $(\mathrm{Fz})$, and its co-receptors, low-density lipoprotein receptorrelated proteins 5 and 6 (LRP5/6), to form a complex $(7,8)$. This complex traps AXN and DVL. As a result, $\beta$-catenin is not degraded and instead accumulates in the cytoplasm (9). Accumulated $\beta$-catenin translocates to the nuclei and binds to the promoter of target genes with T-cell factor/lymphoid enhancer factor (TCF/LEF) (10). In HCC, $\beta$-catenin is mutated and overexpressed, which suggests that the Wnt pathway is constitutively activated (11). Not all HCC cases, however, harbor a mutation of $\beta$-catenin or Axin (6). Hence, targets other than $\beta$-catenin should be investigated in the Wnt pathway. Proliferation of HCC cells is known to be suppressed by short-hairpin RNA of Fz2 (shRNA-Fz2) (12); however, it is not clear whether shRNA-Fz2 suppresses the motility of HCC cells. Another issue is the method of introduction of shRNAFz2. To this end, methods should be developed to introduce shRNA-Fz2 into HCC cells.

Ultrasound (US) generates cavitation bubbles in liquids that eventually collapse (13). When the cavitation bubbles collapse, the nearby cell membrane is rendered porous, and genetic materials or small molecules can enter the cells through these pores (14). This phenomenon is called 'sonoporation'. The strength of the US irradiation is measured in terms of the mechanical index (MI). MI is calculated as the negative peak pressure divided by the square root of the 
frequency (15). US with a higher MI would exhibit stronger biological effects. Plasmids and short interference RNA have been introduced into cultured HCC cells via US irradiation with a diagnostic US device $(16,17)$. One advantage of using diagnostic US is that its safety for the human body has been established. Another advantage is that the irradiation field can be monitored with a display of the US device, which would enable the introduction of therapeutic genes specifically into the target area. One major problem of irradiation with a diagnostic US device is that the efficiency of the introduction of plasmids into cultured cells is low (16).

Microbubbles have conventionally been used to provide a strong contrast of the target area against the surrounding background (18). Microbubbles collapse when irradiated with US, thereby enhancing sonoporation (19). Perfluorobutane microbubbles (Sonazoid ${ }^{\mathrm{TM}}$; Daiichi-Sankyo, Tokyo, Japan) are produced with albumin and are clinically applied to diagnose HCC (20).

With the available background information, in this study, we attempted to evaluate the possibility of suppressing HCC cell motility with shRNA-Fz2. We, furthermore, investigated whether shRNA-Fz2 suppressed the proliferation of HCC cells irradiated with a diagnostic US device, which was enhanced with Sonazoid.

\section{Materials and methods}

Cell culture. Human HCC cell lines, HLF and PLC/PRF/5, were purchased from RIKEN Cell Bank (Tsukuba, Japan). HLF cells and PLC/PRF/5 cells were used for the study because Fz2 is expressed in both these cells (21). The cells were cultured in Dulbecco's modified Eagle's medium (DMEM) (SigmaAldrich, St. Louis, MO, USA) supplemented with $10 \%$ fetal bovine serum (FBS) (Life Technologies, Grand Island, NY, USA). The cells were cultured in 10-cm dishes (Asahi Techno Glass, Funabashi, Japan) in an atmosphere containing $5 \% \mathrm{CO}_{2}$ at $37^{\circ} \mathrm{C}$ in a humidified chamber.

Ultrasound (US) irradiation. Cultured cells or the starchiodide mixture in black 96-well fluoroimmunoassay (FIA) plates (FIA plates) (Greiner Bio-One, Frickenhausen, Germany) were irradiated with US, as illustrated in Fig. 1. The bottom surfaces of the FIA plates consist of a transparent polystyrene film measuring $190 \pm 19 \mu \mathrm{m}$ in thickness. The bottom surfaces of the FIA plates allow the penetration of US to a greater extent than that achieved with the bottom surfaces of other 96-well plates that are relatively thicker. An $8.0-\mathrm{MHz}$ linear-array probe (PLT-805AT; Toshiba Medical Systems, Ohtawara, Japan) was attached to the bottom of the FIA plates (Fig. 1A). US was generated and monitored with SSA-700A (Toshiba Medical Systems). The linear probe covered all the eight wells present in a single row of the FIA plate (Fig. 1B). The FIA plates were irradiated from below for 1 min. The irradiated field was monitored with the display of the SSA-700A (Fig. 1C). MI (0.1, 0.4 and 0.8) was not measured but were actually selected with the US device.

Cell proliferation assay. The cells were trypsinized, harvested, and spread on 96-well plates (Asahi Techno Glass) or FIA plates at a density of 1,000 cells/well. The cells were cultured
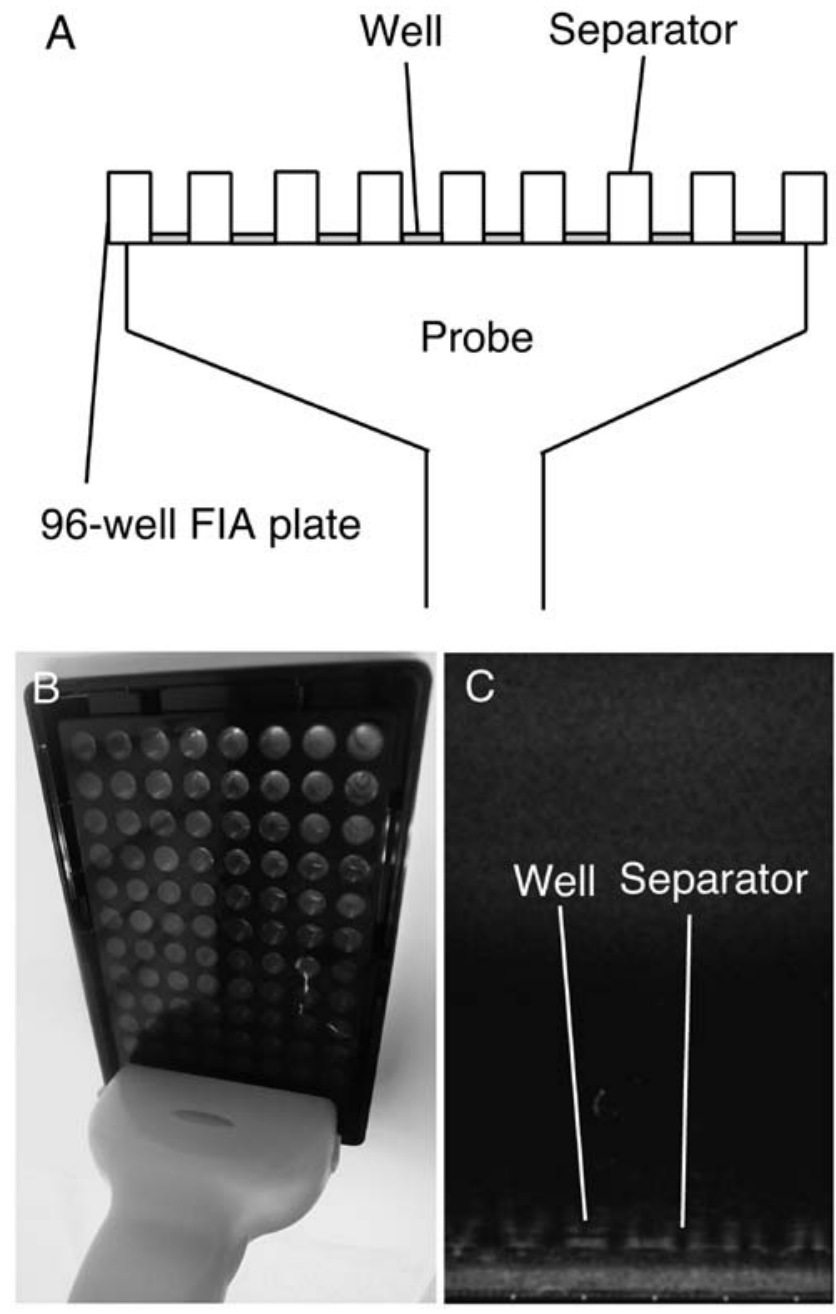

Figure 1. Ultrasound irradiation. A linear-array probe was attached to the bottom of a black 96-well fluoroimmunoassay (FIA) plate (A). The gray box indicates liquid in each well. The length of the probe covered the length of eight wells present in a single row of the FIA plate (B). The irradiation field was monitored with the display of the ultrasound device, SSA-700A.

in DMEM supplemented with $10 \%$ FBS. The cells were transfected with shRNA of Fz2 (OriGene, Rockville, MD, USA) (10 ng or $100 \mathrm{ng}$ in $25 \mu \mathrm{l}$ of Opti-MEM 1 Reduced Serum Media (Life Technologies) per well) by using Lipofectamine LTX (Life Technologies), following manufacturer's instructions. For irradiation with US, $100 \mathrm{ng}$ of shRNA-Fz2 was added in $25 \mu$ of Opti-MEM 1 Reduced Serum Media per well and irradiated with US, as described above. Scrambled shRNA (100 ng/well) was used as a negative control (OriGene). Mock transfection involved carrying out the same transfection procedure with Lipofectamine LTX without using any plasmids. After transfection/irradiation with US, $25 \mu \mathrm{l}$ of DMEM supplemented with 10\% FBS was added to each well. The cells were cultured for $72 \mathrm{~h}$ and subjected to 3-(4,5-dimethylthiazol-2-yl)-5-(3-carboxymethoxyphenyl)2-(4-sulfophenyl)-2H-tetrazolium, inner salt (MTS) assay, according to the manufacturer's instructions (Promega Corp., Madison, WI, USA). MTS is reduced by the cells to a colored formazan product with an absorbance maximum at $490 \mathrm{~nm}$. The absorbance was measured using an iMark Microplate Absorbance Reader (Bio-Rad, Hercules, CA, USA). 
Table I. Primers for real-time quantitative polymerase chain reaction.

\begin{tabular}{|c|c|c|c|c|c|c|}
\hline $\begin{array}{l}\text { Primer } \\
\text { name }\end{array}$ & Sequence & Description & $\begin{array}{l}\text { Product } \\
\text { size (bp) }\end{array}$ & $\begin{array}{l}\text { Annealing } \\
\text { temperature }\end{array}$ & Cycle & $\begin{array}{c}\text { GenBank } \\
\text { accession no. }\end{array}$ \\
\hline OMC355 & 5'- AGAGGCGGAGGAGAACAAACAG-3' & Cyclin D1, forward & 180 & 60 & 40 & NM_053056 \\
\hline OMC356 & 5'-AGGCGGTAGTAGGACAGGAAGTTG-3' & Cyclin D1, reverse & & & & \\
\hline OMC749 & 5'-CCTGGGCAGATTCCAAACCT-3' & MMP9, forward & 89 & 60 & 40 & NM_004994 \\
\hline OMC750 & 5'-GCAAGTCTTCCGAGTAGTTTTGGAT-3' & MMP9, reverse & & & & \\
\hline OMC321 & 5'-CGAATGCCAGAGAAGGTCAC-3' & RPL19, forward & 157 & 60 & 40 & ВC095445 \\
\hline OMC322 & 5'-CCATGAGAATCCGCTTGTTT-3' & RPL19, reverse & & & & \\
\hline
\end{tabular}

MMP9, matrix metalloproteinase 9; RPL19, ribosomal protein L (RPL) 19.

Real-time quantitative polymerase chain reaction. Cells were cultured in DMEM supplemented with 10\% FBS in 6-well plates (Asahi Techno Glass) and transfected with shRNA-Fz2 $(0.25$ or $2.5 \mu \mathrm{g})$ by using Lipofectamine LTX. Scrambled shRNA (2.5 $\mu \mathrm{g} /$ well) was used as a negative control (OriGene). Mock transfection involved carrying out the same transfection procedure with Lipofectamine LTX without using any plasmids. Total RNA (5 $\mu \mathrm{g})$ was isolated with Isogen (Nippon Gene, Tokyo, Japan) and subjected to the synthesis of the first-strand cDNA with SuperScript III and oligo(dT), following the manufacturer's instructions (Life Technologies). Total RNA isolated from an adult liver was purchased from Promega Corp. Real-time quantitative PCR was performed using Fast SYBR Green Master Mix (Life Technologies) with MiniOpticon (Bio-Rad). The results were analyzed using the MiniOpticon system (Bio-Rad). Real-time quantitative PCR was performed for 40 cycles, with $5 \mathrm{sec}$ of denaturation and $5 \mathrm{sec}$ of annealing-extension. Table I shows the primer sequences. RPL19 was used as an internal control, since it is a housekeeping gene that is constitutively expressed (22).

Scratch assay. The cells were plated on 4-well chamber slides (Becton Dickinson, Franklin Lakes, NJ, USA) and scratched with a sterile razor on reaching confluence. Immediately after the scratch was applied, the cells were transfected with shRNA-Fz2 (50 or $500 \mathrm{ng}$ ) by using Lipofectamine LTX. Scrambled shRNA $(2.5 \mu \mathrm{g} /$ well $)$ was used as a negative control (OriGene). Mock transfection involved carrying out the same transfection procedure with Lipofectamine LTX without using any plasmids. After $48 \mathrm{~h}$ of incubation, the cells were subjected to hematoxylin and eosin staining. The slides were observed under an AX80 microscope (Olympus, Tokyo, Japan). The distance between the scratch line and the growing edge of the cell layer was measured at five different points.

Quantification of $\mathrm{H}_{2} \mathrm{O}_{2}$ generation. Generation of $\mathrm{H}_{2} \mathrm{O}_{2}$ was quantified using the starch-iodide method (23). Briefly, $100 \mu \mathrm{l}$ of a mixture of potassium iodide $(0.05 \mathrm{M})$ and starch $(5 \mathrm{mg} / \mathrm{ml})$ was placed into each well of the FIA plate. US irradiation generates $\mathrm{H}_{2} \mathrm{O}_{2}$, and the generated $\mathrm{H}_{2} \mathrm{O}_{2}$ oxidizes I- into I2, which then reacts with starch to form a purple-colored complex. The absorbance of the resulting complex was analyzed at $490 \mathrm{~nm}$ by using an iMark Microplate Absorbance Reader.
Statistical analysis. One-way analysis of variance (ANOVA) was used for statistical analysis with the JMP 10.0.2 software (SAS Institute, Cary, NC). P-values $<0.05$ were determined to be statistically significant.

\section{Results}

As reported in our previous study, shRNA-Fz2 suppresses proliferation of HLF cells (12); however, the experiments were not repeated with HLF cells in the present study. To confirm that PLC/PRF/5 cells expressed Fz2 at higher levels than those in an adult liver, real-time quantitative PCR (qPCR) was performed (Fig. 2A). PLC/PRF/5 cells expressed higher levels of Fz2 than did the adult liver. Next, suppression of the proliferation of PLC/ PRF/5 cells with shRNA-Fz2 was investigated (Fig. 2B). PLC/ $\mathrm{PRF} / 5$ cells were transfected with shRNA-Fz2 and subjected to MTS assay. Cell proliferation was suppressed with shRNAFz2. To confirm that the expression levels of Fz2 were those suppressed by the transfection with shRNA-Fz2, RNA was isolated from the cells and subjected to qPCR after transfection of shRNA-Fz2 into PLC/PRF/5 cells (Fig. 2C). Cyclin D1 is involved in cell proliferation (24); hence, expression levels of cyclin D1 were analyzed with qPCR after transfection of shRNA-Fz2 into PLC/PRF/5 cells (Fig. 2D). The expression levels of Fz2 decreased with the transfection of shRNA-Fz2.

To clarify the suppression of cell motility with shRNAFz2, the scratch assay was performed using HLF cells (Fig. 3A-D) and PLC/PRF/5 cells cultured in 4-well chamber slides (Fig. 3E-H). After the scratch was made with a sterile razor, the cells were transfected with negative control of shRNA at $500 \mathrm{ng} /$ well (Fig. 3B and F), shRNA-Fz2 at $50 \mathrm{ng} /$ well (Fig. 3C and G), or $500 \mathrm{ng} /$ well (Fig. 3D and H). Cells subjected to mock transfection were used as controls (Fig. 3A and E). Distance between the growing edge of the cell layer and the scratch line was measured at five different points for HLF cells (Fig. 3I) and PLC/PRF/5 cells (Fig. 3J). The distances were significantly decreased with transfection of shRNA $(\mathrm{P}<0.05)$.

Matrix metalloproteinase 9 (MMP9) is involved in cell motility (25), and its expression levels increase in HCC tissues (26). The expression levels of MMP9 were therefore, investigated. HLF cells (Fig. 4A) and PLC/PRF/5 cells (B) were transfected with negative control of shRNA 

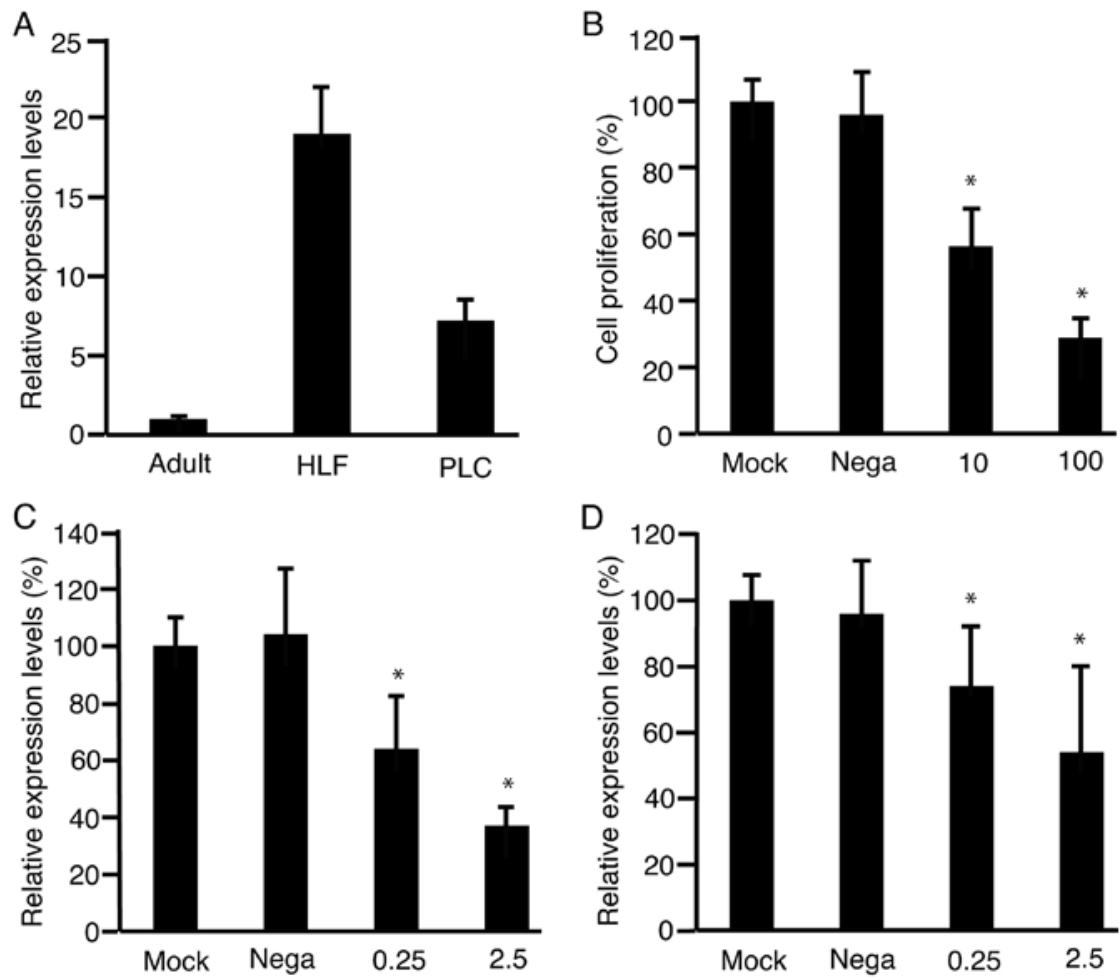

Figure 2. Transfection of short-hairpin RNA of frizzled-2 (shRNA-Fz2) into PLC/PRF/5 cells. Expression levels of frizzled-2 in HLF cells (HLF) and $\mathrm{PLC} / \mathrm{PRF} / 5$ cells (PLC) were analyzed with real-time quantitative PCR (qPCR) as compared with those in the adult liver (adult) (A). After transfection of shRNA-Fz2 at 10 or $100 \mathrm{ng} /$ well in PLC/PRL/5 cells seeded in 96-well plates, cell proliferation was analyzed by the MTS assay (see text) (B). To analyze the expression levels of Fz2 (C) and cyclin D1 (D), qPCR was performed for PLC/PRF/5 cells seeded in 6-well plates that were transfected with 0.25 or $2.5 \mu \mathrm{g} /$ well shRNA-Fz2. Mock, mock transfection; Nega, transfection of negative control of shRNA; 10, $10 \mathrm{ng} / \mathrm{well}$ of shRNA-Fz2 in 96-well plates; 100, $100 \mathrm{ng} / \mathrm{well}$ of shRNA-Fz2 in 96-well plates; 0.25, $0.25 \mu \mathrm{g} /$ well of shRNA-Fz2 in 6-well plates; 2.5, $2.5 \mu \mathrm{g} /$ well of shRNA-Fz2 in 6-well plates; "P<0.05 against Mock, $\mathrm{n}=3$.

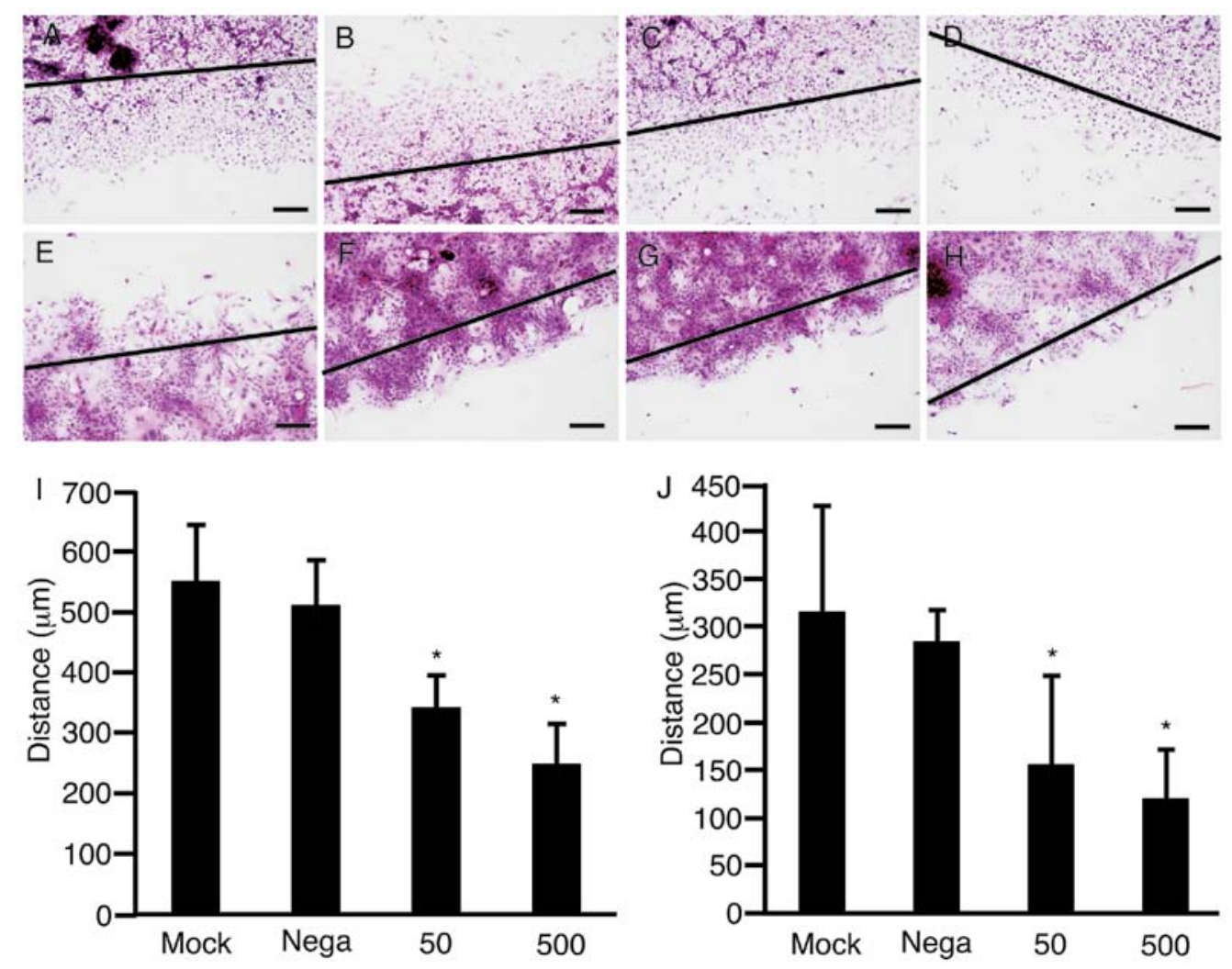

Figure 3. Scratch assay. HLF cells (A-D) and PLC/PRF/5 cells (E-H) were cultured in 4-well chamber slides. After scratching the cell layer with a sterile razor (solid line), negative control of shRNA (B and F) or $50 \mathrm{ng}$ (C and G) or $500 \mathrm{ng}$ (D and H) of shRNA-Fz2 was transfected. The cultured cells were subjected to hematoxylin and eosin staining after $48 \mathrm{~h}$ of culture. The distance between the growing edge of the cell layer and the scratch line was measured at five different points in HLF cells (I) and PLC/PRF/5 cells (J). Original magnification, $\mathrm{x} 100$; scale bar, $200 \mu \mathrm{m}$; ${ }^{*} \mathrm{P}<0.05$ against Mock, $\mathrm{n}=5$. 

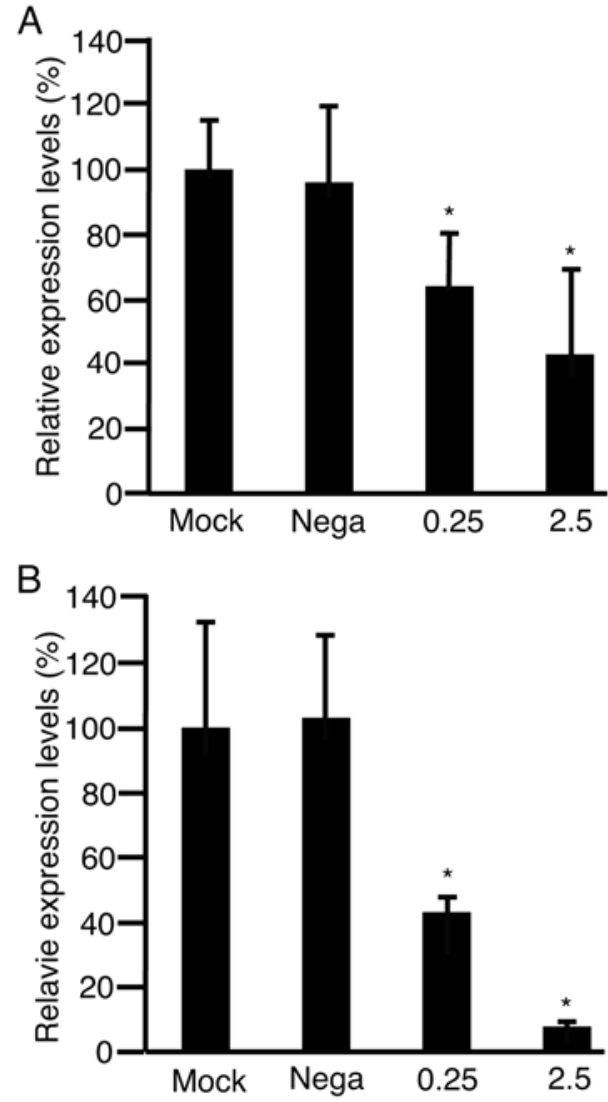

Figure 4. Expression levels of matrix metalloproteinase 9. Short-hairpin RNA of Fz2 [0.25 $\mu \mathrm{g}(0.25)$ or $2.5 \mu \mathrm{g}(2.5)$ each well] was transfected into HLF cells (A) and PLC/PRF/5 cells (B) cultured in 6-well plates. Mock, mock transfection; Nega, negative control of shRNA; "P<0.05 against Mock, $n=3$.

at $2.5 \mu \mathrm{g} /$ well, shRNA-Fz2 at $0.25 \mu \mathrm{g} /$ well, or $2.5 \mu \mathrm{g} / \mathrm{well}$. RNA was isolated from the cells and subjected to qPCR to analyze MMP9 expression after $48 \mathrm{~h}$ culture. The expression levels of MMP9 significantly decreased by transfection with shRNA-Fz2 $(\mathrm{P}<0.05)$.

The above results clearly showed that cell proliferation and motility were suppressed by shRNA-Fz2. It was confirmed that shRNA-Fz2 would be suitable as a therapeutic agent of HCC. The study also aimed to evaluate methods for the introduction of shRNA into HCC cells. Starch-iodide method was applied to detect $\mathrm{H}_{2} \mathrm{O}_{2}$, which was generated as a result of sonoporation (13). Sonazoid was added to the water at $0,1,3,10$ or $30 \%$ in FIA plates after $1 \mathrm{~min}$ of US irradiation MI values of 0.1 (Fig. 5A), 0.4 (B), or 0.8 (C). MI was not monitored but was set on the US device (SSA-700A). Absorbance of the liquid in the well was analyzed at $490 \mathrm{~nm}$. The absorbance significantly decreased with the addition of Sonazoid $(\mathrm{P}<0.05)$. These results suggested that US irradiation with Sonazoid generated $\mathrm{H}_{2} \mathrm{O}_{2}$ and caused sonoporation.

To address the possibility that shRNA-Fz2 suppressed cell proliferation on irradiation with US, HLF cells (Fig. 6A) and PLC/PRF/5 cells (Fig. 6B) were cultured in FIA plates. The cells were irradiated with US at MI values of $0.1,0.4$, or 0.8 after the addition of shRNA-Fz2 (100 ng/well) with or without $30 \%$ Sonazoid. MI was not monitored but was set on the US device. At a MI of 0.4, shRNA-Fz2 suppressed cell proliferation in both cell lines.
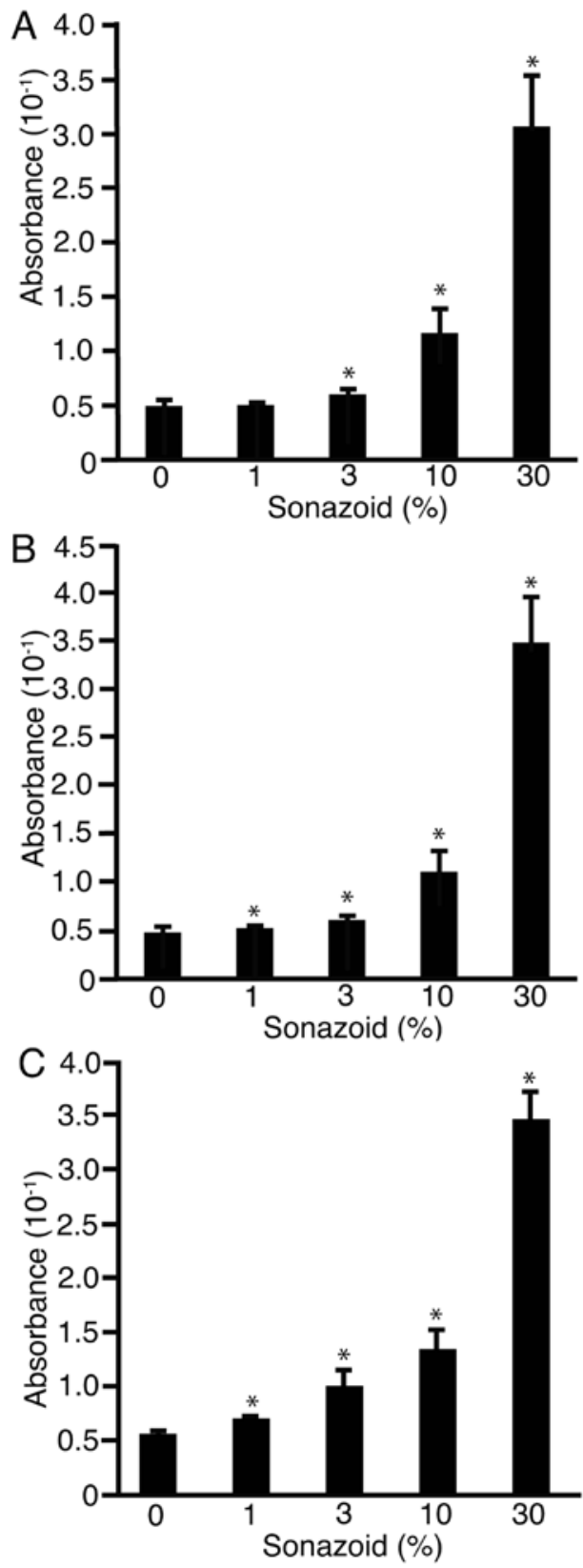

Figure 5. Generation of $\mathrm{H}_{2} \mathrm{O}_{2}$ by ultrasound irradiation with Sonazoid. A $100-\mu 1$ mixture of potassium iodide $(0.05 \mathrm{M})$ and starch $(5 \mathrm{mg} / \mathrm{ml})$ was placed into each well of the FIA plates (see text). Sonazoid was added at 0,1 , 3,10 or $30 \%$ to the mixture. The plates were irradiated with ultrasound from below at a mechanical index of 0.1 (A), 0.4 (B), or 0.8 (C). Absorbance of the mixture was measured. ${ }^{*} \mathrm{P}<0.05$ against $0 \%$.

\section{Discussion}

Prognosis of HCC becomes significantly poorer with metastasis (27). Pathologically, HCC cells invade the surrounding normal tissues, suggesting that the motility of HCC cells is the major factor underlying metastasis (28). Hence, suppression of HCC cell motility is expected to improve the prognosis of HCC. Our results clearly indicated that shRNA-Fz2 suppressed the motility of HLF cells and PLC/PRF/5 cells. Given that our previous report showed that shRNA-Fz2 suppresses HCC cell proliferation (12), the present findings and those of our previous study together show that shRNA-Fz2 is a potential candidate for the treatment of HCC. 

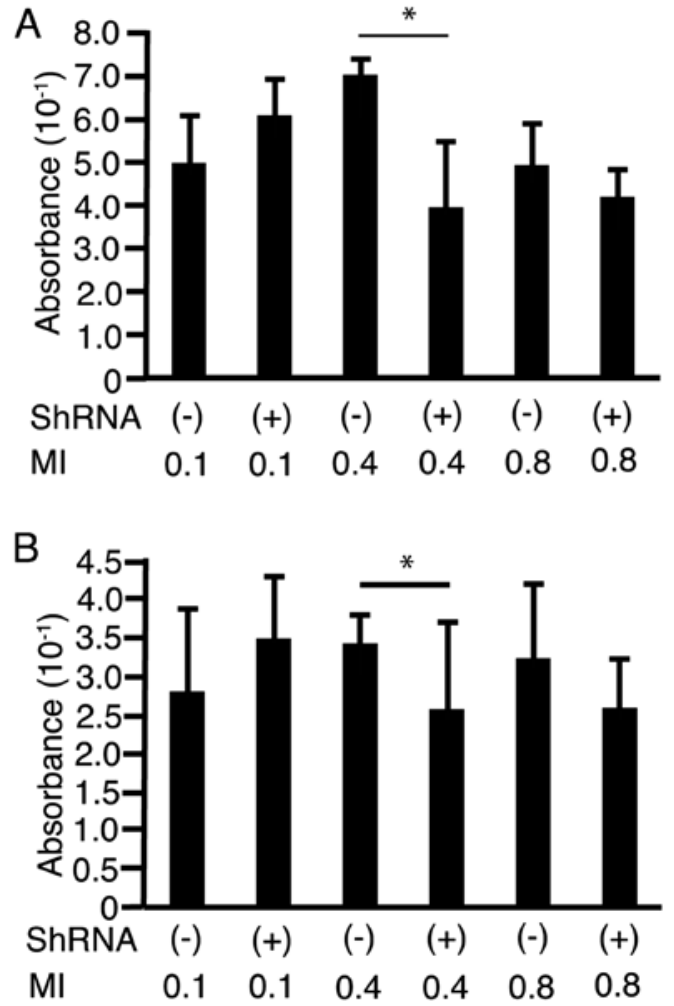

Figure 6. Cell proliferation assay. HLF cells (A) and PLC/PRF/5 cells (B) were cultured in FIA plates (see text). After addition of short hairpin RNA of frizzled-2 (shRNA) at $100 \mathrm{ng} /$ well in $25 \mu \mathrm{l}$ of Opti-MEM 1 Reduced Serum Media with $30 \%$ Sonazoid (+), the cells were irradiated with ultrasound at a mechanical index of $0.1,0.4$, or $0.8(\mathrm{MI})$ for $1 \mathrm{~min}$. Cells that had not been transfected with shRNA were used as negative controls (-). After the irradiation, $25 \mu \mathrm{l}$ of Dulbecco's modified Eagle's medium supplemented with $10 \%$ fetal bovine serum was added. The cells were subjected to MTS assay (see text) after $72 \mathrm{~h}$ of culture. ${ }^{*} \mathrm{P}<0.05$ against shRNA(-) at a mechanical index of 0.4 .

Plasmids can be experimentally introduced into cultured cells or tissues (29); however, the efficiency of this introduction is low, which is a major problem (16). To improve the efficiency, three commercially available microbubbles, SonoVue ${ }^{\mathrm{TM}}$ (Bracco Imaging, Courcouronnes, France), Optison ${ }^{\mathrm{TM}}$ (GE Healthcare, Little Chalfont, UK), and Sonazoid, have been compared for their efficiency in introducing genes into the skeletal muscle (30). The authors report that introduction efficiency does not depend on the size or composition of the microbubbles or the stability of the microbubbles but on their concentration. In the present study, introduction efficiency increased as the concentration of Sonazoid was raised. Sonazoid has been used to introduce plasmids into cultured cells (31); in that study, the authors used a US generator for experimental purposes. In our study, a diagnostic US device was used. Our data showed that introduction of plasmids into cultured cells could be possible with irradiation from a diagnostic US device and by using Sonazoid, which is commercially available.

In the present study, the scratch assay was not performed after US irradiation with the addition of shRNA-Fz2 and Sonazoid, since US did not permeate or weaken the bottom surface of the glass 4-chamber slides. Wells larger than those on FIA plates were not commercially available.

In the future, the strength of US irradiation would be monitored with a hydrophone, and HCC cells would be cultured in larger plates to analyze the expression levels of cyclin D1 and MMP9 after sonoporation with shRNA-Fz2, which was enhanced with Sonazoid.

In conclusion, motility of HLF cells and PLC/PRF/5 cells was suppressed with shRNA-FZ2. Sonazoid enhanced sonoporation mediated by the diagnostic US device and suppression of the proliferation of both cell types with shRNA-Fz2.

\section{References}

1. McGlynn KA, Petrick JL and London WT: Global epidemiology of hepatocellular carcinoma: An emphasis on demographic and regional variability. Clin Liver Dis 19: 223-238, 2015.

2. Knox JJ, Cleary SP and Dawson LA: Localized and systemic approaches to treating hepatocellular carcinoma. J Clin Oncol 33: 1835-1844, 2015.

3. Tejeda-Maldonado J, García-Juárez I, Aguirre-Valadez J, González-Aguirre A, Vilatobá-Chapa M, ArmengolAlonso A, Escobar-Penagos F, Torre A, Sánchez-Ávila JF and Carrillo-Pérez DL: Diagnosis and treatment of hepatocellular carcinoma: An update. World J Hepatol 7: 362-376, 2015.

4. Chen C and Wang G: Mechanisms of hepatocellular carcinoma and challenges and opportunities for molecular targeted therapy. World J Hepatol 7: 1964-1970, 2015.

5. Bogaerts E, Heindryckx F, Vandewynckel YP, Van Grunsven LA and Van Vlierberghe $\mathrm{H}$ : The roles of transforming growth factor- $\beta$, Wnt, Notch and hypoxia on liver progenitor cells in primary liver tumours (Review). Int J Oncol 44: 1015-1022, 2014.

6. Pez F, Lopez A, Kim M, Wands JR, Caron de Fromentel C and Merle P: Wnt signaling and hepatocarcinogenesis: Molecular targets for the development of innovative anticancer drugs. J Hepatol 59: 1107-1117, 2013.

7. Tanaka SS, Kojima Y, Yamaguchi YL, Nishinakamura R and Tam PP: Impact of WNT signaling on tissue lineage differentiation in the early mouse embryo. Dev Growth Differ 53: 843-856, 2011.

8. MacDonald BT, Tamai K and He X: Wnt/beta-catenin signaling: Components, mechanisms, and diseases. Dev Cell 17: 9-26, 2009.

9. Takahashi-Yanaga F: Activator or inhibitor? GSK-3 as a new drug target. Biochem Pharmacol 86: 191-199, 2013.

10. Jamieson C, Sharma M and Henderson BR: Targeting the $\beta$-catenin nuclear transport pathway in cancer. Semin Cancer Biol 27: 20-29, 2014.

11. Rizvi S and Gores GJ: Molecular profiling and research of therapeutic targets. Dig Dis 33: 586-589, 2015.

12. Tomizawa M, Shinozaki F, Motoyoshi Y, Sugiyama T, Yamamoto S and Sueishi M: Short hairpin RNA of frizzled-2 suppresses the proliferation of hepatocellular carcinoma cells. Oncol Lett 8: 1519-1522, 2014.

13. Tomizawa M, Shinozaki F, Motoyoshi Y, Sugiyama T, Yamamoto S and Sueishi M: Sonoporation: Gene transfer using ultrasound. World J Methodol 3: 39-44, 2013.

14. Tomizawa M, Ebara M, Saisho H, Sakiyama S and Tagawa M: Irradiation with ultrasound of low output intensity increased chemosensitivity of subcutaneous solid tumors to an anti-cancer agent. Cancer Lett 173: 31-35, 2001.

15. de Jong N: Mechanical index. Eur J Echocardiogr 3: 73-74, 2002.

16. Tomizawa M, Shinozaki F, Sugiyama T, Yamamoto S, Sueishi M and Yoshida T: Plasmid DNA introduced into cultured cells with diagnostic ultrasound. Oncol Rep 27: 1360-1364, 2012.

17. Tomizawa M, Shinozaki F, Sugiyama T, Yamamoto S, Sueishi M and Yoshida T: Short interference RNA introduced into cultured cells with diagnostic ultrasound. Oncol Rep 27: 65-68, 2012.

18. Salvatore V, Borghi A and Piscaglia F: Contrast-enhanced ultrasound for liver imaging: Recent advances. Curr Pharm Des 18: 2236-2252, 2012.

19. De Cock I, Zagato E, Braeckmans K, Luan Y, de Jong N, De Smedt SC and Lentacker I: Ultrasound and microbubble mediated drug delivery: Acoustic pressure as determinant for uptake via membrane pores or endocytosis. J Control Release 197: 20-28, 2015

20. Claudon M, Dietrich CF, Choi BI, Cosgrove DO, Kudo M, Nolsøe CP, Piscaglia F, Wilson SR, Barr RG, Chammas MC, et al: Guidelines and good clinical practice recommendations for contrast enhanced ultrasound (CEUS) in the liver - update 2012: A WFUMB-EFSUMB initiative in cooperation with representatives of AFSUMB, AIUM, ASUM, FLAUS and ICUS. Ultraschall Med 34: 11-29, 2013. 
21. Fujimoto T, Tomizawa M and Yokosuka O: SiRNA of frizzled-9 suppresses proliferation and motility of hepatoma cells. Int J Oncol 35: 861-866, 2009.

22. Davies B and Fried M: The L19 ribosomal protein gene (RPL19): Gene organization, chromosomal mapping, and novel promoter region. Genomics 25: 372-380, 1995.

23. Kondo T and Yoshii G: Effect of intensity of $1.2 \mathrm{MHz}$ ultrasound on change in DNA synthesis of irradiated mouse L cells. Ultrasound Med Biol 11: 113-119, 1985.

24. Kim JK and Diehl JA: Nuclear cyclin D1: An oncogenic driver in human cancer. J Cell Physiol 220: 292-296, 2009.

25. Vandooren J, Van den Steen PE and Opdenakker G: Biochemistry and molecular biology of gelatinase $\mathrm{B}$ or matrix metalloproteinase-9 (MMP-9): The next decade. Crit Rev Biochem Mol Biol 48: 222-272, 2013

26. Zhang Y, Shen Y, Cao B, Yan A and Ji H: Elevated expression levels of androgen receptors and matrix metalloproteinase-2 and -9 in 30 cases of hepatocellular carcinoma compared with adjacent tissues as predictors of cancer invasion and staging. Exp Ther Med 9: 905-908, 2015.
27. Duseja A: Staging of hepatocellular carcinoma. J Clin Exp Hepatol 4 (Suppl 3): S74-S79, 2014.

28. Tomizawa M, Kondo F and Kondo Y: Growth patterns and interstitial invasion of small hepatocellular carcinoma. Pathol Int 45: 352-358, 1995.

29. Horie S, Watanabe Y, Ono M, Mori S and Kodama T: Evaluation of antitumor effects following tumor necrosis factor- $\alpha$ gene delivery using nanobubbles and ultrasound. Cancer Sci 102: 2082-2089, 2011.

30. Alter J, Sennoga CA, Lopes DM, Eckersley RJ and Wells DJ: Microbubble stability is a major determinant of the efficiency of ultrasound and microbubble mediated in vivo gene transfer. Ultrasound Med Biol 35: 976-984, 2009.

31. Zhang Y, Tachibana R, Okamoto A, Azuma T, Sasaki A, Yoshinaka K, Tei Y, Takagi S and Matsumoto Y: Ultrasoundmediated gene transfection in vitro: Effect of ultrasonic parameters on efficiency and cell viability. Int J Hyperther 28: 290-299, 2012. 